\title{
Accidente lonómico
}

\section{Lonomiasis}

\author{
Albert Alejandro Ávila , Atilio Moreno, Andrés Garzón, \\ Álvaro Andrés Gómez • Bogotá, D.C. (Colombia)
}

\begin{abstract}
Resumen
Se trata de un caso clínico de un adolescente masculino de 15 años de edad remitido del municipio de Arauca-Arauca Colombia por un cuadro clínico de hematuria macroscópica, equimosis y gingivorragia con antecedente de contacto previo con orugas de la familia Saturniidae. Presentaba prolongación de los tiempos de coagulación y disminución del fibrinógeno. El diagnóstico fue establecido por la anamnesis, las manifestaciones clínicas y los reportes de laboratorio. Las medidas terapéuticas tuvieron éxito. Las características fisiopatológicas y clínicas del accidente lonómico serán revisadas. (Acta Med Colomb 2013; 38: 95-100)
\end{abstract}

Palabras clave: lepidópteros, fibrinólisis, larva, Colombia.

\section{Abstract}

This is a case report of a 15 years old male teenager referred from the municipality of Arauca, Arauca Colombia by a clinical picture of macroscopic hematuria, bruising and gingivorrhagia with a history of previous contact with caterpillars of the family Saturniidae. Clotting times were prolonged and fibrinogen was decreased. The diagnosis was established by history, clinical manifestations and laboratory reports. Therapeutic measures were successful. Pathophysiological and clinical characteristics of the lonimia accident have been reviewed. (Acta Med Colomb 2013; 38: 95-100)

Keywords: lepidoptera, fibrinolysis, larva, Colombia.

\begin{abstract}
Dr. Albert Alejandro Ávila Álvarez: Residente de Medicina de Urgencias, Pontificia Universidad Javeriana, Hospital Universitario San Ignacio; Dr. Atilio Moreno Carrillo: Medicina Interna P.U.J. Advanced Fellowship in Emergency Medicine G.W.U. Director Programa de Especialización Medicina de Urgencias, Pontificia Universidad Javeriana, Hospital Universitario San Ignacio; Dres. Andrés Garzón Forero y Álvaro Andrés Gómez Venegas: Residentes de Medicina Interna. Pontificia Universidad Javeriana, Hospital Universitario San Ignacio, Bogotá, D.C. (Colombia).

Correspondencia. Dr. Albert Alejandro Ávila Álvarez

E-mail: alejandroavila2010@gmail.com Recibido: 29/IV/2012 Aceptado: 17/V/2013
\end{abstract}

\section{Introducción}

El envenenamiento por orugas pertenecientes al género lepidóptera incluye los siguientes síndromes: erucismo, lepidopterismo, dendrolimiasis, oftalmia nodosa y coagulopatía de consumo con fibrinólisis secundaria. En el accidente lonómico los síndromes descritos son el erucismo y la coagulopatía de consumo con fibrinólisis secundaria (1-5). El erucismo por Saturniidae es el envenenamiento resultante del contacto con orugas en la etapa larval de la especie Lonomia (Figura 1. B). Se estima que más de 250000 especies de lepidópteras habitan la tierra, de las cuales unas 180000 han sido descritas hasta ahora $(1,3)$. Los lepidópteros están divididos en 4 subórdenes: Zeugloptera, Aglossata, Heterobathmiina y Glossata $(1,2)$, con aproximadamente 100 familias, de las cuales 12 son de importancia médica $(3,5,6)$. La familia Saturniidae perteneciente al suborden Glossata incluye 26 especies de lonomia que se encuentran distribuidas en el continente americano (1, 2,7-9). Sin embargo, sólo Lonomia obliqua y Lonomia achelous causan accidentes graves.

L. achelous se encuentra principalmente en Venezuela y la Guyana Francesa $(10,11)$ y existen reportes en Paraguay,
Perú y Ecuador. Otra variedad se encuentra en el norte de Brasil, donde se clasifica como $L$. diabolous $(3,10)$. $L$. obliqua se encuentra en el sur y sureste de Brasil (12-16). L. obliqua también se encuentra en Uruguay, Paraguay y Argentina (3). Donde los casos reportados por accidente lonómico arrojan una incidencia de ocho casos por cada 100.000 habitantes (17).

En Colombia se han reportado casos por L. descimoni, $L$. rufescens, $L$. claucina, $L$. occidentalis, $L$. armata, $L$. inexpectata y L. Saturniidae. De los casos reportados provenientes de los departamentos del Casanare y de la Amazonia se ha identificado como responsable a L. achelous (18).

La importancia del accidente lonómico no radica únicamente en su incidencia, sino en las tasas de morbilidad y mortalidad asociadas (19). En las últimas décadas algunas especies están adquiriendo importancia por la gravedad de los casos y su expansión geográfica. Representantes del género lonomia están migrando a otras zonas en las que no se hallaban, desplazándose desde las áreas rurales a las áreas urbanas debido a la deforestación, colonización, eliminación de predadores naturales y al cambio climático global $(4,20,21)$. 


\section{Presentación del caso}

Paciente masculino de 15 años de edad, procedente y remitido del hospital local de Arauca, Arauca (Colombia), quien ingresa al servicio de urgencias del Hospital Universitario San Ignacio de Bogotá -HUSI, por presentar un cuadro clínico de cinco días de evolución, que inicia con el contacto en la palma izquierda con orugas que se encontraban en el tallo de un árbol. Presentando dolor de intensidad moderada en el sitio de contacto. Dos días después presenta sangrado en laceraciones recientes de miembros inferiores, hematuria, gingivorragia y equimosis. Consulta al hospital local de Arauca, Arauca donde toman paraclínicos que evidencian prolongación en los tiempos de coagulación e inician manejo médico con la administración endovenosa de una unidad de plasma fresco congelado. Ante la persistencia de la prolongación de los tiempos de coagulación de etiología desconocida deciden remitir.

A su ingreso al HUSI, se encontró a un paciente con los siguientes signos vitales: TA $110 / 60 \mathrm{mmHg}$, frecuencia cardiaca de $72 \mathrm{lpm}$, frecuencia respiratoria de $16 \mathrm{rpm}$ y saturación de oxígeno de $96 \%$, mucosas húmedas, con estigmas de gingivorragia. A la auscultación ruidos cardiacos rítmicos sin soplos, ruidos respiratorios normales. En la mano izquierda presentaba edema y calor local de forma difusa. Además de un hematoma en cara interna del muslo y pierna izquierda y varios hematomas pequeños en extremidades superiores e inferiores. El examen neurológico era normal.

Se comienza hidratación con cristaloides endovenosos y se solicitan paraclínicos con los siguientes resultados:

TP $>120$ segundos, TPT $>180$ segundos, INR $>$ de 11 , Fibrinógeno $<60 \mathrm{mg} / \mathrm{dL}$ : hemoglobina: 13,5g/dL, hematocrito: $39.2 \%$, plaquetas: 212000 .

Uroanálisis: hemoglobina $1000 \mathrm{xc}$, hematíes incontables.

BUN $10,7 \mathrm{mg} / \mathrm{dL}$, creatinina $0,87 \mathrm{mg} / \mathrm{dL}$, sodio $141,4 \mathrm{mmol} / \mathrm{L}$, potasio $4,06 \mathrm{mmol} / \mathrm{L}$.

Se consideró que el paciente presentaba un accidente lonómico con una coagulopatía secundaria asociada. Dado que los casos de los últimos años en el país involucran a
L. achelous, que posee un veneno con un mecanismo de predominio fibrinolítico asociado al consumo de factores de coagulación, y ante la ausencia del suero antilonómico en el país se inicia el manejo con ácido tranexámico 500 $\mathrm{mg} / \mathrm{IV} / 6 \mathrm{~h}$ y complejo protrombínico 1800 unidades intravenoso $(30 \mathrm{mg} / \mathrm{kg}$ ) (48). El control de paraclínicos demostraba persistencia en la prolongación de los tiempos de coagulación con consumo del fibrinógeno. Al siguiente día de su ingreso se logra la consecución por parte del ministerio de la Protección Social de Colombia de cinco ampollas de suero antilonómico que se administraron inmediatamente (48). Treinta minutos después de la infusión el paciente comienza a presentar lesiones maculopapulares pruriginosas de $4 \mathrm{~mm}$ en el tórax $(47,48,53)$, que ceden con la aplicación de hidrocortisona $300 \mathrm{mg}$ y difenhidramina $50 \mathrm{mg}$ endovenosos. Se complementa el manejo con la aplicación de 10 unidades de crioprecipitados $(47,48)$.

Posterior a la aplicación del suero antilonómico el paciente comienza a presentar corrección progresiva de los tiempos de coagulación. Cuatro días después de observación, los controles de paraclínicos se encontraban dentro de límites normales y se da de alta (Tabla 2).

\section{Discusión}

El contacto con la oruga suele ocurrir de forma accidental ya que las orugas se agrupan en los tallos y ramas de los árboles cercanos a las residencias (Figura 1.A) $(3,7)$. La oruga de lonomia tiene cerdas de color verde pardusco de diferentes tamaños en el cuerpo, éstas son evaginaciones duras y espinosas de la cutícula y están presentes en todas las etapas larvales $(16,17,22)$. Las cerdas tienen en el extremo distal una punta fina rica en quitina que se rompe fácilmente para liberar el veneno presente en el canal hueco interno que lo almacena (22).

La gravedad del envenenamiento se relaciona principalmente con el contacto con una colonia que contiene al menos $40-50$ orugas. Cada oruga produce aproximadamente 2.56 $\mathrm{mg}$ de veneno $(4,17-22)$.

Tabla 2. Evolucion en el tiempo de los paraclinicos.

\begin{tabular}{|c|c|c|c|c|c|c|c|c|}
\hline Fecha & $31 / 05 / 2011$ & $01 / 06 / 2011$ & $03 / 06 / 2011$ & $04 / 06 / 2011$ & 05/06/2011 & 06/06/2011 & $07 / 06 / 2011$ & 0/06/2011 \\
\hline $\mathrm{Hb} \mathrm{g} / \mathrm{dL}$ & 1.9 & & 13.0 & 12.5 & 10.8 & 11.7 & & 12.6 \\
\hline Plaquetas & 199000 & & 212000 & 181000 & 183000 & 205000 & & 265000 \\
\hline $\mathrm{TP}$ & $>3$ minutos & $>2$ minutos & $>2$ minutos & 24 segundos & 13 segundos & 12 segundos & 12 segundos & 12 segundos \\
\hline TPT & $>3$ minutos & $>2$ minutos & $>3$ minutos & 41 segundos & 24 segundos & 23 segundos & 23 segundos & 26 segundos \\
\hline INR & & & $>11$ & 2.1 & 1.2 & 1.1 & 1.1 & 1.1 \\
\hline Fibrinógeno $\mathrm{mg} / \mathrm{dL}$ & & & $<60$ & Indetectable & 123 & 129 & 184 & 184 \\
\hline \multicolumn{9}{|c|}{ Complejo protrombínico + Ácido tranexamico } \\
\hline
\end{tabular}




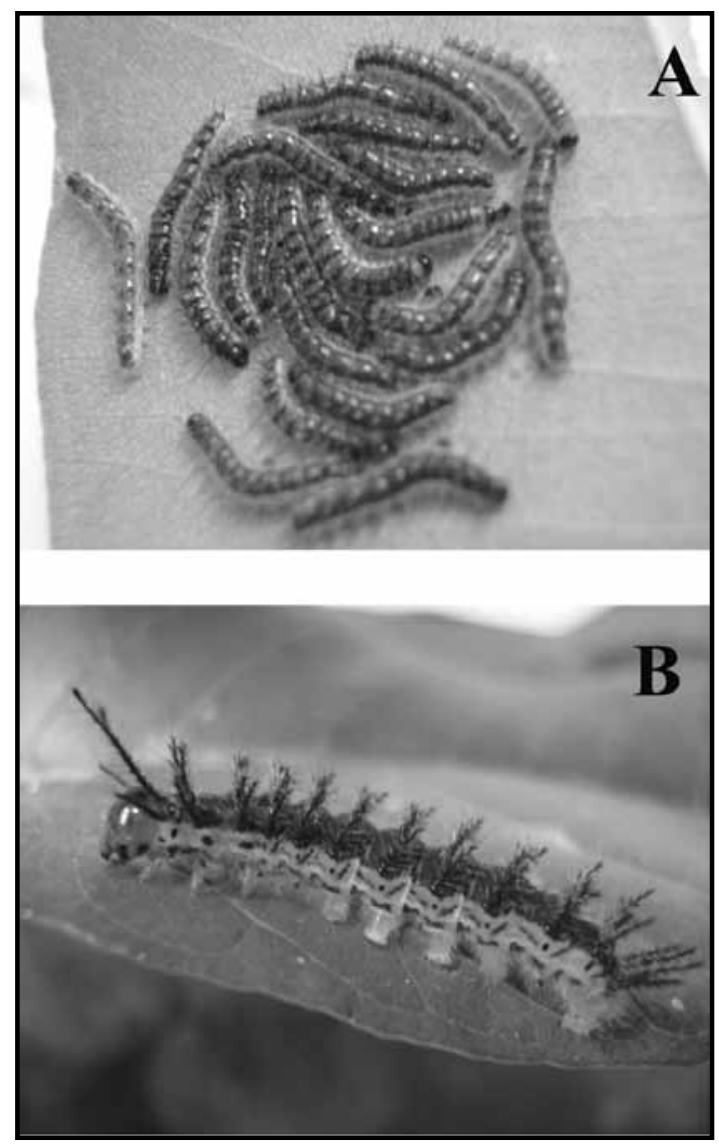

Figura 1. (Lepidóptera: Saturniidae). A: larvas en tercer estado larvario. B: larva en séptimo estado larvario.

Características del veneno. Los venenos de las especies L. obliqua y L. achelous tienen efectos similares, pero los mecanismos por el que causan sus efectos son diferentes (23).

Veneno de L. Obliqua. Está compuesto por toxinas entre las que se encuentran factores activadores del factor de coagulación II, del factor X, $\alpha$-fibrogenasas, hialuronidasas, lipocalinas, serpinas y fosfolipasa A2 (22, 24-30).

El veneno de L. obliqua provoca una forma especial de coagulación intravascular diseminada (CID) con coagulopatía de consumo, agotamiento de los factores V y XIII, así como fibrinólisis secundaria $(15,17,29-32)$. Esto es diferente de CID observada en otras situaciones clínicas, como traumatismo, neoplasia o sepsis $(4,17,26,29,33-37)$.

De las cerdas de L. obliqua se han extraído dos toxinas procoagulantes: un activador del factor $\mathrm{X}$, llamado losac (factor activador Stuart de L. obliqua) y un activador de la protrombina llamado lopap (proteasa activadora de protrombina de lonomia obliqua). Lopap realiza un papel importante en el síndrome hemorrágico inducido por contacto con la oruga L. oblicua $(26,29,35,37-39)$.

Toxinas procoagulantes. 1. Lopap, es una serinoproteasa de $69 \mathrm{kDa}$, activadora de protrombina independiente de los cofactores de protrombinasa (Factor V activado, fosfolípidos).
Los estudios in vivo apoyan la hipótesis de que lopap a través de la generación de trombina contribuye a la presentación de la coagulopatía de consumo. La fragmentación de la protrombina por lopap es similar a la inducida por el factor $\mathrm{X}$ activado, en ausencia de protrombinasa, formando pretrombina 2 , fragmentos $1+2$ y trombina, sin formación de meizotrombina $(39,40)$.

Lopap estimula el aumento de la interleuquina 8, de la molécula de adhesión intercelular-1(ICAM-1) y selectina E, así como también aumenta la secreción de óxido nítrico (NO) y prostaglandinas (PGI 2). El NO puede participar por varios mecanismos $(15,31,34)$ : puede inducir daño en los tejidos al generar peroxinitritos y radicales hidroxilo, contribuyendo a la hipotensión, a la inhibición de la agregación plaquetaria, a la inhibición del fosfoinositol 3 quinasa (PI3K) y a la supresión de la vía del flujo de calcio intracelular. También puede suprimir la expresión de selectina $\mathrm{P}$ y el cambio conformacional de la glicoproteína IIb-IIIa (GPIIb-IIIa) (31). En modelos in vitro se ha podido demostrar que en el envenenamiento por L. obliqua la disminución de la agregación plaquetaria coincide con la elevación de los niveles séricos de NO, sobre todo después de seis horas, momento en que se observa la máxima inhibición $(31,33,34,41)$.

2. Losac, es una serino-proteasa de $43 \mathrm{kDa}$. Capaz de activar el Factor X de una forma dependiente de la concentración. Losac no activa otros factores de coagulación (15).

Trombocitopenia. Se detecta en algunos de los pacientes, sobre todo en los casos más graves $(17,42)$. La agregación plaquetaria disminuye seis horas después de la exposición al veneno. Alteración que se acompaña de la prolongación de los tiempos de coagulación. Las manifestaciones hemorrágicas tales como gingivorragia o sangrado de las heridas indican que hay alteración de la adhesión plaquetaria. Los estudios in vivo evidencian una reducción de aproximadamente $26-30 \%$ en el recuento plaquetario a las seis horas, normalizándose después de las 24 horas. El consumo de plaquetas no parece ocurrir, ya que sólo hay reducción leve en su número $(17,22,43,44)$. Probablemente, la inhibición de la agregación puede prevenir el consumo de plaquetas y la formación de trombos en los lechos vasculares. Por lo tanto, la disminución de la agregación puede contribuir a la presentación del síndrome hemorrágico observado en el envenenamiento por L. obliqua $(22,42,43)$.

Hemólisis intravascular. El veneno de L. obliqua provoca hemólisis intravascular en ratas, con una reducción en el número de eritrocitos circulantes, aumento de la hemoglobina plasmática en las primeras seis horas y una disminución en los niveles de la hemoglobina total a las 24 horas $(37,43$, 45). La hemólisis intravascular se ha observado sólo en los casos severos que tienen como antecedente el contacto con un gran número de orugas $(46,47)$. Se cree que la responsable de la acción hemolítica es la fosfolipasa A2 (43), enzima que induce actividad hemolítica exclusivamente indirecta y responsable de provocar reacciones locales, como el dolor y la inflamación $(17,22,30,39)$. 
Veneno de $\boldsymbol{L}$. Achelous. El contacto con orugas de $L$. achelous produce síndrome fibrinolítico. Pruebas de laboratorio realizadas en los pacientes muestran una intensa actividad fibrinolítica. El veneno y las fracciones fibrinolíticas degradan la fibrina in vitro. Sin embargo, los productos de la degradación obtenidos son completamente diferentes de la plasmina o productos de la degradación de tripsina. El veneno degrada los coágulos preformados a un ritmo más lento que la plasmina. Además, los inhibidores de la proteasa del plasma del sistema fibrinolítico ( $\alpha 2$-antiplasmina, $\alpha$ 2-macroglobulina) sólo inhiben débilmente el efecto lítico del veneno $(47,48)$.

El veneno de L. achelous tiene actividad tanto procoagulante como anticoagulante. Las principales toxinas del veneno de $L$. achelous son lonomin II, que tiene actividad fibrinolítica directa, lonomin III, que es un activador de protrombina, lonomin IV, que actúa como activador del Factor X, lonomin V, enzima proteolítica que ejerce su actividad sobre el zimógeno del Factor XIII activo y sobre sus subunidades A y B, inactivándolo, y también tiene actividad similar a la uroquinasa ( activando el plasminogeno), lonomin VI:a y lonomin VI:i, que actúan como activadores o inhibidores del Factor V. Lonomin VII, tiene actividad similar a la calicreína y a lonomin I, achelasa I y achelasa
II tienen acción fibrinolítica similares a las de la plasmina (11, 47-49) (Figura 2).

Signos y síntomas. Los síntomas clínicos por el envenenamiento de L. obliqua son muy similares a los observados por L. achelous. Estos síntomas y signos inician con dolor local, eritema y edema en la zona de contacto, posteriormente aparece cefalea, náuseas y vómito. Seis a 72 horas después del envenenamiento puede aparecer hipotensión, hematomas, hematuria, equimosis, epistaxis, gingivorragia, sangrado de heridas recientes y melenas(4, 41-43, 47-49).

El tipo de reacción depende de la especie de la oruga involucrada, el grado de exposición, el estadio de desarrollo del lepidóptero y de las características del paciente. Los síntomas los podemos clasificar como leves, moderados o severos (Tabla 1). Las complicaciones potencialmente mortales incluyen insuficiencia renal aguda (IRA) y hemorragias intracerebrales, alveolares o peritoneales (15-40-45-50-52).

La incoagulabilidad de la sangre se demuestra por la significativa prolongación de los parámetros hemostáticos, como el tiempo de protrombina (TP), el tiempo de tromboplastina parcial activado (TPT), tiempo de trombina (TT), y trastornos asociados con el consumo de factores de la coagulación, principalmente el consumo del fibrinógeno así como los factores V, XIII, Proteína C y antitrombina III. Los

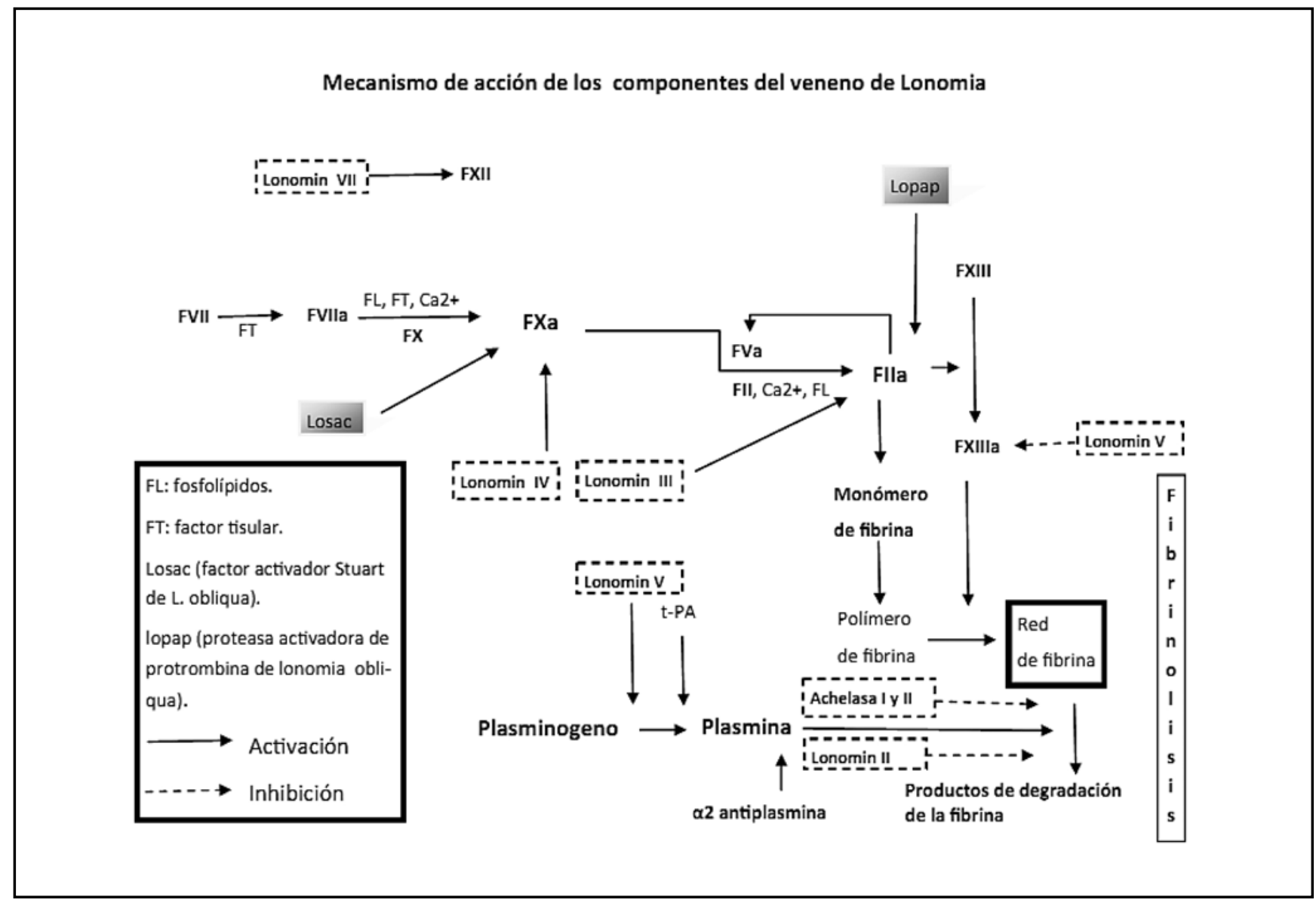

Figura 2. Mecanismo de acción de los componentes del veneno de lonomia. 
Tabla 1. Grados de severidad.

\begin{tabular}{|l|l|l|l|}
\hline Severidad & Leve & Moderado & Severo \\
\hline Manifestaciones locales & $\begin{array}{l}\text { Presentes } \\
\text { o Ausentes }\end{array}$ & $\begin{array}{l}\text { Presentes } \\
\text { o Ausentes }\end{array}$ & Presentes \\
\hline Tiempos de Coagulación & Normales & Prolongados & Prolongados \\
\hline $\begin{array}{l}\text { Manifestaciones } \\
\text { Hemorrágicas }\end{array}$ & Ausentes & $\begin{array}{l}\text { Hematomas } \\
\text { o Equimosis } \\
\text { o Hematuria }\end{array}$ & Parenquimatosas \\
\hline Insuficiencia Renal Aguda & Ausente & $\begin{array}{l}\text { Presente } \\
\text { o Ausente }\end{array}$ & Presente \\
\hline
\end{tabular}

factores VIII:c, von Willebrand, productos de degradación de fibrina y el dímero D se encuentran elevados. El factor II esta disminuido en casos severos (16, 24, 21, 47, 53).

La IRA se observa en 2-5\% de los pacientes después del contacto con L. obliqua y $10 \%$ de éstos puede desarrollar enfermedad renal crónica $(6,34,51)$. Los pacientes con IRA presentan una mayor frecuencia de hematuria y grandes cambios en los parámetros de coagulación. Sin embargo, la patogénesis de las complicaciones renales es desconocida. Cambios hemodinámicos, isquemia renal y la acción directa de las toxinas del veneno pueden contribuir a esta patogénesis $(6,51)$.

La IRA es más frecuente después del contacto con $L$. obliqua que con L. achelous.

Tratamiento. El tratamiento con la terapia convencional con fármacos antifibrinolíticos como la aprotinina 400000 KIU en una hora seguido de 200000 KIU cada seis horas en infusión continua, el ácido épsilon aminocaproico 20 gramos día (dosis iniciales de $30 \mathrm{mg} / \mathrm{kg}$ por vía endovenosa continuando con $15 \mathrm{mg} / \mathrm{kg} / 4 \mathrm{~h}$ ), o el ácido tranexámico $15 \mathrm{~g} / \mathrm{d}$ (oral o endovenoso) se han utilizado con éxito para tratar a los pacientes envenenados por L. achelous. La administración de fibrinógeno purificado o los crioprecipitados 4-8 U/24 h (48), contribuyen a la corrección de los tiempos de coagulación. Por el contrario, la terapia de reemplazo, ya sea con sangre total o plasma fresco congelado ha demostrado que pueden agravar los síntomas clínicos del envenenamiento.

La administración de ácido épsilon aminocaproico, para revertir las manifestaciones clínicas de los pacientes envenenados por L. obliqua no permite ni la recuperación del fibrinógeno, ni la normalización de los parámetros hemostáticos, pero sí aumenta la tasa de mortalidad (54).

En el envenenamiento por L. obliqua el suero antilonómico es el único tratamiento efectivo, ya que obtiene la recuperación del fibrinógeno y la normalización de los tiempos de protrombina (TP) y tiempo parcial de tromboplastina activado (TPT), independiente del tiempo de la administración $(6,14,30,54)$. Los antifibrinolíticos no deben ser administrados y la administración de san- gre total o plasma fresco congelado no está indicada, ya que exacerban la coagulación intravascular diseminada $(42,51,54)$.

\section{Conclusión}

Los accidentes con orugas de las especies lonomia cada vez son más frecuentes. Lo que probablemente esté relacionado con el desequilibrio ecológico constante causado por la deforestación, el avance de la frontera agrícola, la colonización y el calentamiento global.

Este reporte reafirma la importancia de la historia clínica y examen físico para el establecimiento del diagnóstico. Sin el antecedente obtenido del contacto previo con una oruga de la familia Saturniidae en una ciudad donde no se presentan estos accidentes, la posibilidad de retraso en el diagnóstico y en el tratamiento es alta. Así mismo se advierte la posibilidad de remisiones de nuevos casos a la red hospitalaria de la ciudad de Bogotá y se alerta sobre el ecoturismo que ha aumentado las tasas de accidentes en los parques de reserva natural. Los viajeros a zonas endémicas generalmente pertenecen a las ciudades principales del país lo que hace necesario que el personal de salud de zonas no endémicas esté preparado para la atención de este tipo de envenenamiento.

\section{Refererencias}

1. Wolff M. Insectos de Colombia. 1ra Ed. Medellín-Colombia: Multimpresos Ltda. 2006: 333-365.

2. Amat G,Andrade MG, Amat E. 1ra Ed. libro rojo de los invertebrados terrestres de Colombia. Bogotá: instituto de ciencias naturales. Universidad nacional de Colombia. Instituto Alexander Von Humboldt, Ministerio de ambiente, vivienda y desarrollo territorial. 2007: 107-143.

3. De Roodt AR, Salomon OD, Orduna TA. Accidents due to lepidoptera with special reference to Lonomia sp. Medicina 2000; 60(6): 964-972.

4. Ramos AT, Goncalves LR, Ribeiro OG, Rocha Campos AC, Sant'Anna OA. Effects of Lonomia obliqua (lepidoptera, saturniidae) toxin on clotting, inflammatory and antibody responsiveness in genetically selected lines of mice. Toxicon 2004; 43(7): 761-768.

5. Diaz JH. The evolving global epidemiology, syndromic classification, management, and prevention of caterpillar envenoming. Am J Trop Med Hyg 2005 72(3): 347-57.

6. Gamborgi GP, Metcalf EB, Barros EJ. Acute renal failure provoked by toxin from caterpillars of the species Lonomia obliqua. Toxicon 2006; 47(1): 68-74.

7. Madrigal A. Insectos forestales en Colombia. 1ra Ed. Medellín-Colombia: MarinVieco Ltda. 2003: pag.312

8. Metcalf CL, Flint WP. Insectos destructivos e insectos útiles, sus costumbres y su control. 1ra Ed. Mexico D.F-Mexico: compañía editorial continental Ltda.1976: 280-305.

9. Coll-Sangrona E,Arocha-Pinango CL. Fibrinolytic action on fresh human clots of whole body extracts and two semipurified fractions from Lonomia achelous caterpillar. Braz J Med Biol Res 1998; 31(6): 779-784.

10. Carrijo-Carvalho LC, Chudzinski-Tavassi AM. The venom of the Lonomia caterpillar: an overview. Toxicon 2007; 49(6): 741-757.

11. Hommel D, Bouchareine L, Hulin A. Acute poisoning by the caterpillar Lonomia achelous. A review of the literature. Report of two cases in French Guyana. Sem Hop Paris 1996; 71(1): 9-12.

12. Correa MS, Siqueira-Batista R, Gomes AP, Franco-Barbosa A, Verzola AC, Oliveira FR, et al. Lonomia erucism in Teresopolis, Rio de Janeiro State, Brazil: report of a probable case and review. Rev Soc Bras Med Trop 2004, 37(5): 418-421.

13. Garcia CM, Danni-Oliveira IM. Occurrence of accidents caused by Lonomia obliqua Walker, in the State of Parana between 1989 and 2001. Rev Soc Bras Med Trop 2007; 40(2): 242-246.

14. Donato J.L, Moreno R.A, Hyslop S, Duarte A, Antunes E, Le Bonniec B.F, 
Rendu, F, de Nucci G. Lonomia oblique caterpillar spicules trigger human blood coagulation via activation of factor $\mathrm{X}$ and prothrombin. Thrombosis and Haemostasis 1998; 79(3): 539-542.

15. Duarte AC, Crusius PS, Pires CA, Schilling MA, Fan HW. Intracerebralhaemorrhage after contact with Lonomia caterpillars. Lancet 1996; 348(9033): 1033.

16. Lisete ML. Aspectos Morfológicos de Lonomia obliqua Walker (Lepidoptera: Saturniidae). Neotropical Entomology 2001; 30(3): 373-379.

17. Pineda D, Amarillo A, Becerra J, Montenegro G. Sindrome hemorrágico por contacto con orugas del género lonomia (saturniidae) en casanare, colombia: informe de dos casos. Biomédica 2001; 21: 328-32.

18. Gamborgi GP, Metcalf EB, Barros EJ. Acute renal failure provoked by toxin from caterpillars of the species Lonomia obliqua. Toxicon 2006; 47(1): 68-74.

19. Wolff JL, Moraes RH, Kitajima E, de Souza Leal E, de A Zanotto PM. Identification and characterization of a baculovirus from Lonomia obliqua (Lepidoptera: Saturniidae). J Invertebr Pathol 2002; 79(3): 137-45.

20. McConnell J. 14th International Conference on Infectious Diseases. Lancet Infect Dis 2010; 10(5): 301-301.

21. Veiga AB, Blochtein B, Guimaraes JA. Structures involved in production, secretion and injection of the venom produced by the caterpillar Lonomia obliqua (Lepidoptera, Saturniidae). Toxicon 2001; 39(9): 1343-1351.

22. Fritzen M, Schattner M, Ribeiro AL, Batista IF, Ventura J, Prezoto BC, et al. Lonomia obliqua venom action on fibrinolytic system. Thromb Res 2003; 112(1-2): $105-110$.

23. da C B Gouveia AI, da Silveira RB, Nader HB, Dietrich CP, Gremski W, Veiga SS. Identification and partial characterisation of hyaluronidases in Lonomia obliqua venom. Toxicon 2005; 45(4): 403-10

24. Pinto AF, Berger M, ReckJ,Jr, Terra RM, Guimaraes JA. Lonomia obliqua venom: In vivo effects and molecular aspects associated with the hemorrhagic syndrome. Toxicon 2010; 56(7): 1103-1112.

25. Pinto AF, Dobrovolski R, Veiga AB, Guimaraes JA. Lonofibrase, a novel alpha-fibrinogenasefrom Lonomia obliqua caterpillars. Thromb Res 2004; 113(2): 147-154.

26. Pinto AF, Dragulev B, Guimaraes JA, Fox JW. Novel perspectives on the pathogenesis of Lonomia obliqua caterpillar envenomation based on assessment of host response by gene expression analysis. Toxicon 2008; 51(6): 1119-1128.

27. Pinto AF, Silva KR, Guimaraes JA. Proteases from Lonomia obliqua venomous secretions: comparison of procoagulant, fibrin(ogen)olytic and amidolytic activities. Toxicon 2006; 47(1): 113-121.

28. Prezoto BC, Maffei FH, Mattar L, Chudzinski-Tavassi AM, Curi PR. Antithrombotic effect of Lonomia obliqua caterpillar bristle extract on experimental venous thrombosis. Braz J Med Biol Res 2002; 35(6): 703-712.

29. Veiga AB, Pinto AF, Guimaraes JA. Fibrinogenolytic and procoagulant activities in the hemorrhagic syndrome caused by Lonomia obliqua caterpillars. Thromb Res 2003; 111(1-2): 95-101.

30. Berger M, ReckJ,Jr, Terra RM, Beys da Silva WO, Santi L, Pinto AF, et al. Lonomia obliqua venomous secretion induces human platelet adhesion and aggregation. J Thromb Thrombolysis 2010; 30(3): 300-310.

31. Fry BG, Roelants K, Champagne DE, Scheib H, Tyndall JD, King GF, et al. The toxicogenomic multiverse: convergent recruitment of proteins into animal venoms. Annu Rev Genomics Hum Genet 2009; 10: 483-511.

32. Reis CV, Kelen EM, Farsky SH, Portaro FC, Sampaio CA, Fernandes BL, et al. A Ca++ activated serine protease (LOPAP) could be responsible for the haemorrhagic syndrome caused by the caterpillar Lonomia obliqua. L obliqua Prothrombin Activator Protease. Lancet 1999; 353(9168): 1942.

33. Berger M, ReckJ,Jr, Terra RM, Pinto AF, Termignoni C, Guimaraes JA. Lonomia obliqua caterpillar envenomation causes platelet hypoaggregation and blood incoagulability in rats. Toxicon 2010; 55(1): 33-44

34. Riella MC, Chula D, de Freitas S, Mazza MM, Pachaly MA. Acute renal failure and haemorrhagic syndrome secondary to toxin of caterpillars (Lonomia obliqua). NDT Plus 2008; 1(6): 445-446.

35. Rubio GB. Epidemiological surveillance of distribution of the caterpillar Lonomia obliqua Walker, 1855, in the State of Parana, Brazil. Cad Saude Publica 2001; 17(4): 1036.
36. Seibert CS, Oliveira MR, Goncalves LR, Santoro ML, Sano-Martins IS Intravascular hemolysis induced by Lonomia obliqua caterpillar bristle extract: an experimental model of envenomation in rats. Toxicon 2004; 44(7): 793-799.

37. Goncalves LR, Sousa-e-Silva MC, Tomy SC, Sano-Martins IS. Efficacy of serum therapy on the treatment of rats experimentally envenomed by bristle extract of the caterpillar Lonomia obliqua: comparison with epsilon-aminocaproic acid therapy. Toxicon 2007; 50(3): 349-356.

38. Seibert CS, Tanaka-Azevedo AM, Santoro ML, Mackessy SP, SoaresTorquato RJ, Lebrun I, et al. Purification of a phospholipase A2 from Lonomia obliqua caterpillar bristle extract. Biochem Biophys Res Commun 2006; 342(4): 1027-1033.

39. Kowacs PA, Cardoso J, Entres M, Novak EM, Werneck LC. Fatal intracerebral hemorrhage secondary to Lonomia obliqua caterpillar envenoming: case report. Arq Neuropsiquiatr 2006; 64(4): 1030-1032.

40. Chudzinski-Tavassi AM, Alvarez Flores MP. Exploring new molecules and activities from Lonomia obliqua caterpillars. Pathophysiol Haemost Thromb 2005; 34(4-5): 228-233

41. Zannin M, Lourenço DM, Motta G, Dalla Costa LR, Grando M, Gamborgi GP, et al. Blood coagulation and fibrinolytic factors in 105 patients with hemorrhagic syndrome caused by accidental contact with Lonomia obliqua caterpillar in Santa Catarina, southern Brazil. Thromb Haemost 2003; 89(2): 355-64.

42. Seibert CS, Santoro ML, Tambourgi DV, Sampaio SC, Takahashi HK, Peres CM, et al. Lonomia obliqua (Lepidoptera, Saturniidae) caterpillar bristle extract induces direct lysis by cleaving erythrocyte membrane glycoproteins. Toxicon 2010; 55(7): 1323-1330.

43. De Castro Bastos L, Veiga A.B, Guimara es J.A, Tonussi C.R. Nociceptive and edematogenic responses elicited by a crude bristle extract of Lonomia obliqua caterpillars. Toxicon 2004; 43: 273-278.

44. Seibert CS, Shinohara EM, Sano-Martins IS. In vitro hemolytic activity of Lonomia obliqua caterpillar bristle extract on human and Wistar rat erythrocytes. Toxicon 2003; 41(7): 831-839.

45. Malaque C,Andrade L, Madalosso G, Tomy S, Tavares FL, Seguro AC. Short report: a case of hemolysis resulting from contact with a Lonomia caterpillar in southern Brazil. Am J Trop Med Hyg 2006; 74: 807-809.

46. Lucena S, Salazar AM, Gil A, Arocha-Pinango CL, Guerrero B. The action of Lonomin V (Lonomia achelous) on fibronectin functional properties. Thromb Res 2008; 121(5): 653-661.

47. Arocha-Piñango CL, Guerrero B. Síndrome hemorrágico producido por contacto con orugas: estudios clínicos y experimentales. Invest Clin 2003; 44(2): $155-163$.

48. Coll-Sangrona E,Arocha-Piñango CL. Fibrinolytic action on fresh human clots of whole body extracts and two semipurified fractions from Lonomia achelous caterpillar. Braz J Med Biol Res 1998; 31(6): 779-84.

49. Bohrer CB, Reck Junior J, Fernandes D, Sordi R, Guimaraes JA, Assreuy J, et al. Kallikrein-kinin system activation by Lonomia obliqua caterpillar bristles: involvement in edema and hypotension responses to envenomation. Toxicon 2007; 49(5): 663-669.

50. Kowacs PA, Cardoso J, Entres M, Novak EM, Werneck LC. Fatal intracerebral hemorrhage secondary to Lonomia obliqua caterpillar envenoming: case report. Arq Neuropsiquiatr 2006; 64(4): 1030-2.

51. Burdmann EA, Antunes I, Saldanha LB, Abdulkader RC. Severe acute renal failure induced by the venom of Lonomia caterpillars. Clin Nephrol 1996; 46(5): 337-9.

52. da Silva GH, Hyslop S, Alice da Cruz-Hofling M. Lonomia obliqua caterpillar venom increases permeability of the blood-brain barrier in rats. Toxicon 2004 44(6): 625-634.

53. Arocha-Piñango C.L, de Bosch N.B, Torres A, Goldstein C, Nouel A, Arguello A, Carvajal Z, Guerrero B, Ojeda A, Rodriguez A. Six new cases of a caterpillar-induced bleeding syndrome. Thrombosis and Haemostasis 1992; 67(4): 402-407.

54. Bogdanov V.Y, Balasubramanian V, Hathcock J, Vele O, Lieb M, Nemerson Y. Alternatively spliced human tissue factor: a circulating, soluble, thrombogenic protein. Nat Med 2003; 9 (4): 458-462. 